UCRL-JC-119359

PREPRINT

\title{
TRANSPORT OF A PARTIALLY-NEUTRALIZED ION BEAM IN A HEAVY-ION FUSION REACTOR CHAMBER
}

\author{
Debra A. Callahan and A. Bruce Langdon
}

\section{Lawrence Livermore National Laboratory, University of California P.O. Box 5508, L-440, Livermore California 94550, USA}

This paper was prepared for submittal to the Proceedings of the IEEE 1995 Particle Accelerator Conference

Dallas, Texas

May 1-5, 1995

April 25, 1995

This is a preprint of a paper intended for publication in a journal or proceedings. Since changes may be made before publication, this preprint is made available with the understanding that it will not be cited or reproduced without the permission of the author.

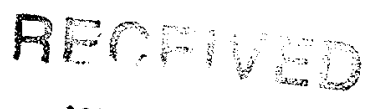
JUL 25 1995

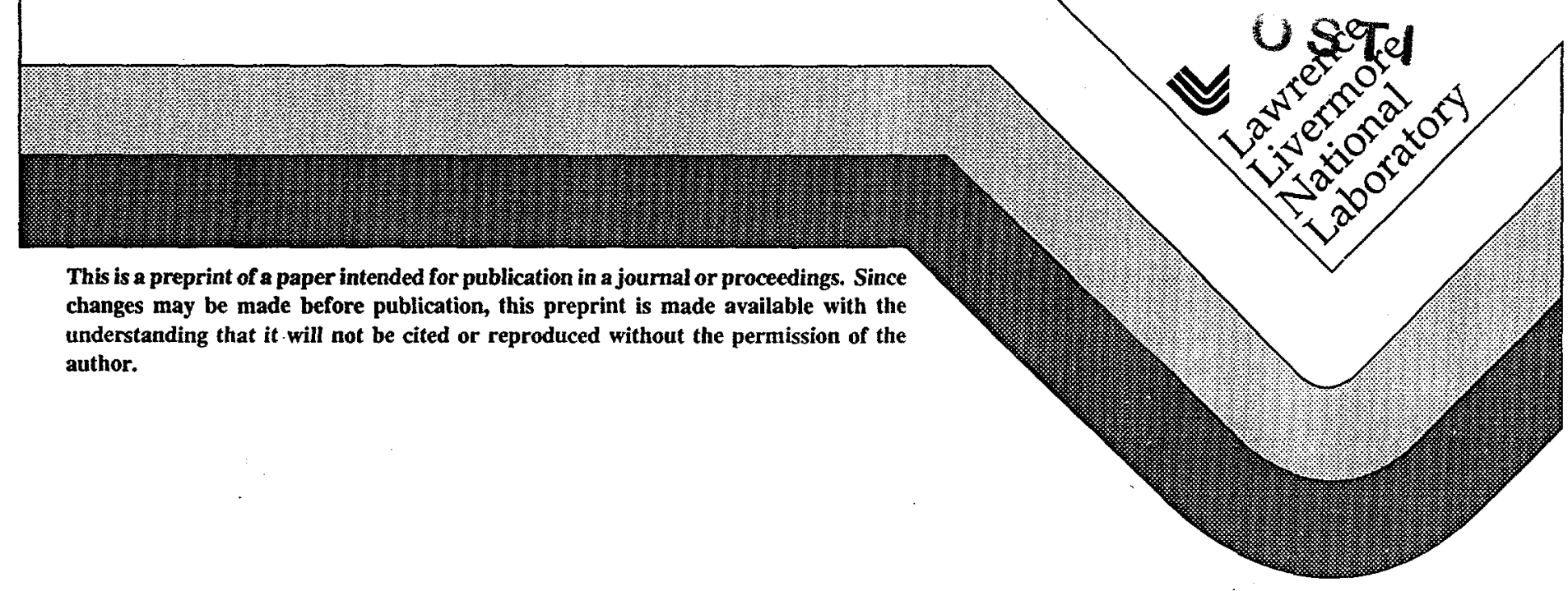




\section{DISCLAIMER}

Portions of this document may be illegible in electronic image products. Images are produced from the best available original document. 


\title{
TRANSPORT OF A PARTIALLY-NEUTRALIZED ION BEAM IN A HEAVY-ION FUSION REACTOR CHAMBER*
}

\author{
Debra A. Callahan and A. Bruce Langdon \\ Lawrence Livermore National Laboratory, Livermore, CA 94550 USA
}

\begin{abstract}
In a heavy-ion driven, inertial confinement fusion power plant, a space-charge dominated beam of heavy ions must be transported through a reactor chamber and focused on a 2-3 $\mathrm{mm}$ spot at the target. The spot size at the target is determined by the beam emittance and space charge, plus chromatic aberrations in the focusing lens system and errors in aiming the beam. The gain of the ICF capsule depends on the focal spot size. We are investigating low density, nearly-ballistic transport using an electromagnetic, $r-z$ particle-in-cell code. Even at low density $\left(n \approx 5 \times 10^{13}\right.$ $\mathrm{cm}^{-3}$ ), beam stripping may be important. To offset the effects of stripping and reduce the space charge, the beam is partially charge neutralized via a pre-formed plasma near the chamber entrance. Additional electrons for charge neutralization come from ionization of the background gas by the beam. Simulations have shown that stripping can greatly increase the spot size; however, partial neutralization can offset most of this increase.
\end{abstract}

\section{INTRODUCTION}

In a heavy ion fusion (HIF) reactor, a space-charge dominated beams of heavy ions must be focused and transported through the reactor chamber and hit a $2-3 \mathrm{~mm}$ spot on the inertial confinement fusion (ICF) target. The gain of the target is a function of the spot size that can be achieved with the beams and ultimately sets the requirements on allowable beam quality in the accelerator.

The beam spot size at the target is influenced by four factors. The beam's transverse emittance and space charge, chromatic aberrations in the final focusing magnet system, and errors in aiming the beam at the target. Generally, the spot size at the target should be approximately,

$$
\begin{aligned}
\left(r_{\text {target }}\right)^{2} \approx & \left(r_{\text {space charge +emittance }}\right)^{2} \\
& +\left(r_{\text {chromatic aberrations }}\right)^{2}+\left(r_{\text {aiming }}\right)^{2}
\end{aligned}
$$

where $r_{\text {target }}$ is the final spot size at the target and $r_{x}$ is the spot size from effect $x$. We strive to achieve $r_{\text {target }}=2-3$ $\mathrm{mm}$. The target is injected into the chamber by an air gun and estimates of the aiming error give $r_{\text {aiming }}=.4 \mathrm{~mm}[1]$. A final design for the final focus system is still needed. We assume $r_{\text {chromatic aberrations }} \approx 1-1.5 \mathrm{~mm}$. For a $3 \mathrm{~mm}$ spot, $r_{\text {space charge +emittance }} \leq 2.8 \mathrm{~mm}$.

Our studies have revolved around low chamber density, nearly-ballistic transport. We feel that this is a more conservative option than pinched or channel transport

\footnotetext{
*Work performed under the auspices of the U.S. DoE by LLNL under contract W-7405-ENG-48.
}

schemes. Pinched and channel transport schemes could drastically reduce the requirements on beam quality and the cost of electricity. The physics of these schemes is not well understood, however. An understanding of the simpler, nearly-ballistic transport will give the HIF program at least one credible scheme for chamber transport.

\section{THE BICrz PARTICLE-IN-CELL CODE}

The BICrz (Beam In Chamber) code was written to study HIF chamber transport. BICrz is an axisymmetric, electromagnetic particle-in-cell code. Beam ions and energetic electrons are modeled as particles to preserve kinetic effects. The code includes a model for stripping of the beam ions by the background gas and collisional ionization of the background gas molecules by the beam ions.

BICrz uses a nearly orthogonal grid which converges with the beam. In a typical chamber design, the beam radius decreases from $5 \mathrm{~cm}$ to $3 \mathrm{~mm}$ over a distance of 3 meters. The converging grid concentrates resolution near the target where it is needed.

\section{HYLIFE-II REACTOR DESIGN}

Our simulations are based on the HYLIFE-II reactor design[2]. In HYLIFE-II, the $4 \mathrm{MJ}$ of energy in the main pulse is supplied by 10 beam of mass $200 \mathrm{amu}$, charge state +1 ions. Each beam carries $4 \mathrm{kA}$ current and has a $10 \mathrm{nsec}$ pulse duration. Two additional beams are used to generate the prepulse ("foot") required by the target. The chamber first wall is located 3 meters from the target. Each beam has a $1^{\circ}$ convergence angle.

The chamber wall is protected by a liquid salt, $\left(\mathrm{BeF}_{2}\right)(\mathrm{LiF})_{2}$, "Flibe." Moir's studies[3] show that $.5 \mathrm{~m}$ of liquid Flibe (density $2 \mathrm{~g} / \mathrm{cc}$ ) will protect the structural material from neutron damage for the full 30 year lifetime of a power plant. In addition, a thickness of $.8 \mathrm{~m}$ of liquid Flibe will allow for shallow burial of 304 stainless steel upon decommissioning of the plant.

The chamber gas will be composed of $\mathrm{BeF}_{2}$ and $\mathrm{LiF}$ molecules. At the temperatures of interest for the HYLIFE-II reactor $\left(600-700^{\circ} \mathrm{C}\right)$, the gas will be rich in $\mathrm{BeF}_{2}$ by about a factor of 10 over LiF. For $650^{\circ}$, the density of the chamber gas will be about $5 \times 10^{13} \mathrm{~cm}^{-3}$.

Our simulations use a $10 \mathrm{GeV}$, mass 210 , charge state +1 beam of heavy ions. The beam has a parabolic current profile with a maximum current of $4.688 \mathrm{kA}$ and duration of $8 \mathrm{nsec}$. This beam is shorter than that used in the reactor $(10$ nsec) for computational convenience. The shorter beam requires fewer particles which makes the calculation faster. With these beam parameters, vacuum transport 
will produce a reasonable spot size at the target. We hope to extend the nearly-ballistic transport mode to the density of the HYLIFE-II chamber.

\section{VACUUM TRANSPORT}

Simulations of beam transport in vacuum can be compared with the beam envelope equation to verify the code. Figure (1) shows the beam as the head reaches 1,2 , and 3 meters along with the envelope solution. Ballistically transporting the particles forward, we find the distance at which the root-mean-square radius is the smallest. For the particles shown in figure (1), the best focus occurs at 2.82 $\mathrm{m}$ with $95 \%$ of the particles falling inside a $2.6 \mathrm{~mm}$ radius. The envelope solution agrees well with the simulation and predicts the best focus at 2.83 meters with a spot size of $2.7 \mathrm{~mm}$.

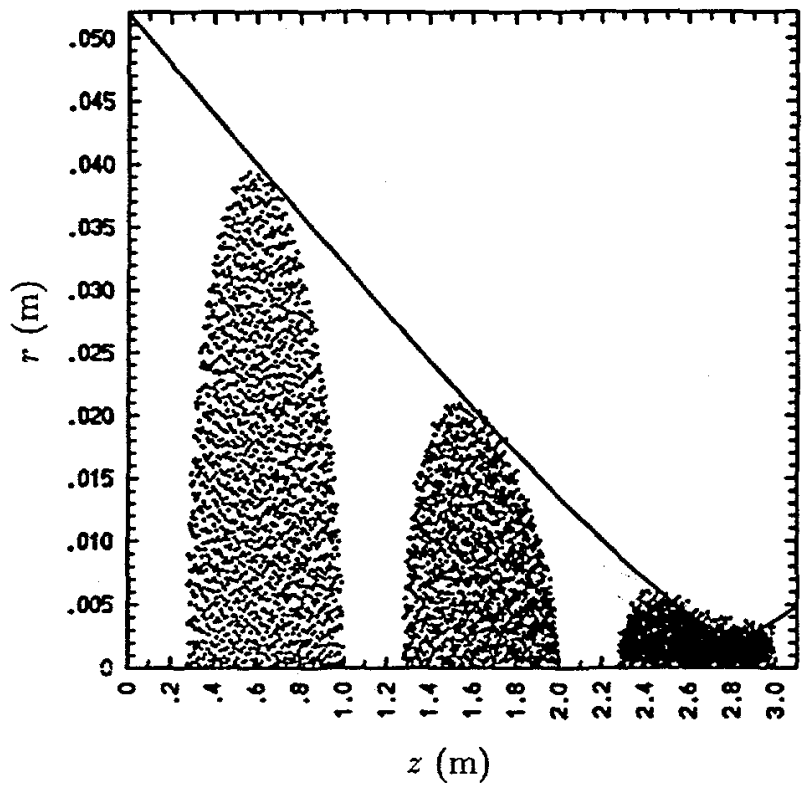

Figure. 1. Beam at 3 times and envelope solution for vacuum transport

These simulations used a normalized emittance of $10.4 \pi$ mm-mrad. In a 3 meter chamber, this results in a spot size of $2 \mathrm{~mm}$ from emittance alone. This value of the emittance was chosen for computational convenience because it prevents the beam, and hence the cell size, from becoming too small. To achieve the small spots needed in a reactor, an emittance of about half this value is necessary.

\section{BEAM STRIPPING AND GAS IONIZATION}

Even at the low densities of the HYLIFE-II chamber, it appears that beam stripping will be important. Cross sections for $10 \mathrm{GeV}, \mathrm{Pb}^{+1}$ ions and $\mathrm{BeF}_{2}$ gas have been difficult to obtain. Estimates of the cross section range from $1.2-4 \times 10^{-16} \mathrm{~cm}^{2}[4],[5],[6]$ and leads to a range of meanfree-paths (MFPs) of $0.5-1.6 \mathrm{~m}$ at $650^{\circ} \mathrm{C}$. These MFPs are smaller than the chamber radius ( 3 meters) so stripping will play a role in understanding beam propagation.
While beam stripping makes chamber propagation more difficult, ionization of the background gas by the beam can partially neutralize the beam and aid transport. Cross sections for collisional ionization of $\mathrm{BeF}_{2}$ by the beam have a larger uncertainty than stripping cross sections because calculating molecular cross sections is more difficult than calculating atomic cross sections. Estimates of the MFP for ionizing the background gas range from $0.7 \mathrm{~m}$ to 25 $\mathrm{m}[4],[8],[7]$.

Knowing the ratio of these cross sections would be helpful because there is some flexibility in the chamber density. Reducing the temperature from $650^{\circ}$ to $600^{\circ} \mathrm{C}$ reduces the density by a factor of 4 .

\section{A. Beam Stripping and Gas Ionization Sensitivity Study}

Because there is uncertainty in the cross sections, we did a series of simulations to understand how these processes effect the beam spot size at the target.

Currently, BICrz does not contain an actual target. Instead, we run the simulation until the beam head reaches the distance of best focus. The particles are then ballistically transported to this same distance and the radius which contains $95 \%$ of the charge is found. This radius is then the spot size.

We found a stripping MFP of 1.2 meters increased the spot size from $2.6 \mathrm{~mm}$ (vacuum transport) to $8 \mathrm{~mm}$. Using the same stripping MFP, but adding a background gas ionization MFP of $3.0 \mathrm{~m}$ reduced the spot size from $8 \mathrm{~mm}$ to $5.4 \mathrm{~mm}$. A gas ionization MFP of $.4 \mathrm{~m}$ (which is outside the range of MFPs discussed in the previous section) reduces the spot size from $8 \mathrm{~mm}$ to $3.7 \mathrm{~mm}$.

We found that adding electrons to the system causes the radial electric field to become nonlinear. Figures (1) and (2) show the radial velocity deflection of particles near the beam center as a function of radius for an initially cold beam. In figure (2), there are no electrons present and the field is linear with radius. Figure (3) includes electrons from both beam stripping and collisional ionization and the field is clearly not linear. In our focusing system, we can compensate for a linear electric field by increasing the focusing angle, but we cannot compensate for the nonlinear fields in this same way. In addition, the ions have a velocity spread because they are stripped at different times. Once the ion strips, it responds more strongly to the electric fields.

\section{NEUTRALIZATION VIA A PREFORMED PLASMA ANNULUS}

Neutralizing an ion beam is more difficult than neutralizing an electron beam. If an electron beam passes through a plasma, the plasma electrons are moved out of the beam path by the beam's self field and leave the immobile plasma ions behind to neutralize the beam. In the case of an ion beam, electrons must be pulled in from outside the beam path in order to neutralize.

An electron produced by ionizing the background gas only reduces the net charge after the beam has left the 


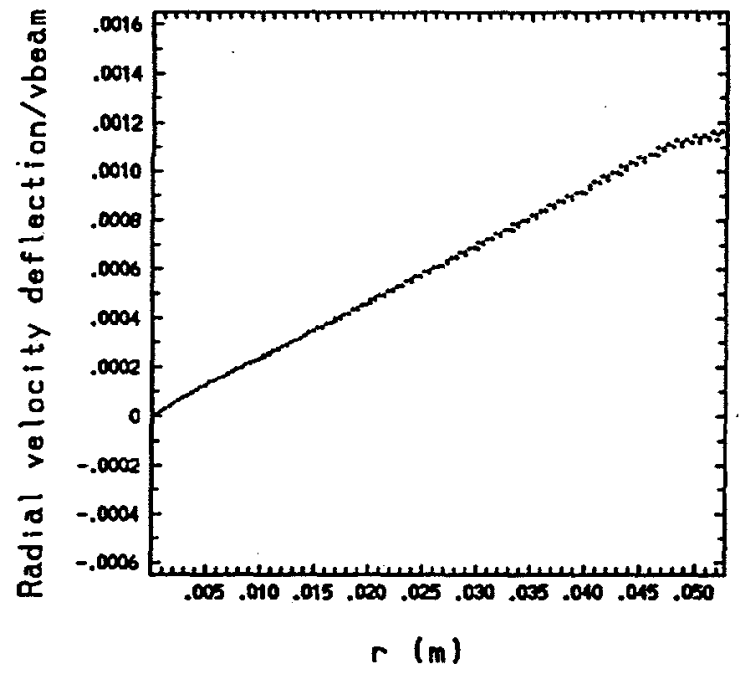

Figure. 2. In vacuum transport, the radial electric field is linear. We overfocus the beam to compensate for these linear fields.

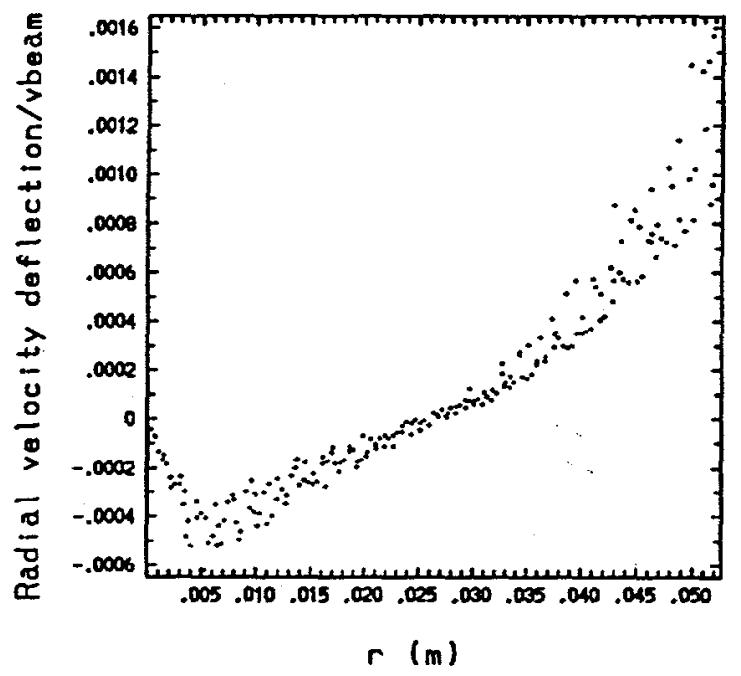

Figure. 3. When electrons are added (from beam stripping and gas ionization in this case), the radial field becomes nonlinear. We cannot compensate for the nonlinear field by simply overfocusing the beam.

gas ion behind. As a result, gas ionization neutralizes the beam very slowly and radial velocities develop before neutralization takes place. These radial velocities result in an increase in spot size at the target.

To neutralize the beam quickly, we added a preformed plasma annulus near the chamber entrance. As the beam enters the chamber, it pulls in electrons from the annulus and leaves a positively charged plasma behind. We found that this was quite effective at neutralizing the beam initially. However, the entrained electrons are hot $\left(v_{t h} \approx .3 c\right)$ and do not compress as readily as the beam ions. Hence, neutralization is not as good near the target.

Our simulations used an annulus that was . 3 meters long placed $5 \mathrm{~cm}$ inside the chamber entrance. The annulus was designed to fit between the beams in HYLIFE-II and had total electron charge of 4 times the beam charge. Adding the annulus to a simulation with a stripping MFP of $1.2 \mathrm{~m}$ decreased the spot size from $8 \mathrm{~mm}$ to $3.5 \mathrm{~mm}$. Adding gas ionization should improve the spot size because the electrons produced by gas ionization are not as hot as the electrons from the plasma annulus and will aid neutralization near the target. Simulations confirm this prediction and the spot size with the plasma annulus plus a gas ionization MFP of 3 meters results in a spot size of $3.0 \mathrm{~mm}$.

\section{CONCLUSIONS}

Although some uncertainty remains in the cross section, beam stripping seems important at the gas density of the HYLIFE-II chamber. Simulations have shown that stripping can increase the spot size considerably. Stripping causes the beam ions to respond more strongly to any electric fields present. In addition, electrons in the chamber cause nonlinear electric fields which cannot be compensated for by the final focusing system.

Charge neutralization of the beam by both background gas ionization and a preformed plasma annulus have been studied. Neutralization via gas ionization happens slowly since the beam must ionize the gas molecule, then leave the resulting ion behind before the net charge is reduced. The spot size is reduced with gas ionization, but radial velocities develop before neutralization can occur.

A preformed plasma annulus placed near the chamber entrance is quite effective at neutralizing the beam early on. The beam pulls in electrons from the annulus quickly, before radial velocities develop. However, these electrons are hot, and become less effective at neutralizing as the beam compresses near the target. The best spot size was found with a combination of gas ionization and a preformed plasma annulus.

Future studies will include ionizing a larger volume of the background gas surrounding the entire beam path. We hope that this will result in better charge neutralization which may allow the use of higher current beams. Higher current beams, in the form of either multiply charged ions or lower energy, lighter ions (Cs for example), should reduce the cost of a heavy ion driver considerably; however, high current beams must be partially charge neutralized in the chamber even in vacuum.

\section{References}

[1] R. W. Petzold, PhD Thesis, U.C. Davis, 1995.

[2] R. W. Moir, et al., Fusion Technology, 25, 5 (1994).

[3] R. W. Moir, Nuclear Engineering and Design, 29, 34 (1995).

[4] N. Barboza, U.C. Berkeley, Private Comm. (1994).

[5] W. R. Meier, et al., "Osiris and Sombrero Inertial Confinement Fusion Power Plant Designs," WJSA-92-01, DOE/ER/54100-1 (1992).

[6] B. Badger, et al., "HIBALL-2, An Improved Conceptual Heavy Ion Beam Driven Fusion Reactor Study," UWFDM-625, U. of Wisconsin (1984).

[7] R. W. Moir, LLNL, Private Comm. (1994).

[8] W. Ping, U. of Wisconsin, Private Comm. (1994). 